Supporting Information for:

\title{
Osmotic Pressure Simulations of Amino Acids and Peptides Highlight Potential Routes to Protein Force Field Parameterization
}

\author{
Mark S. Miller, Wesley K. Lay and Adrian H. Elcock* \\ Department of Biochemistry, University of Iowa, Iowa City, IA 52242 \\ e-mail: adrian-elcock@uiowa.edu \\ telephone: 3193356643
}




\section{Supplementary Figures}

Figure S1 Example Simulation System. Simulation box containing 0.5 M Phe (spheres) in TIP3P water (red dots) with box dimensions $4.8 \times 4.8 \times 9.6 \mathrm{~nm}$. Solute molecules were restrained to the central region of the box by a flat-bottomed potential (gray walls), which were used to report the osmotic pressure.

Figure S2 Simulations Compared to Previous Work. A. Comparison of rational osmotic coefficients from simulations of Gly modeled using the KBFF in SPC/E from this work (yellow) and by the Smith group (Black, ref. 28). B. Comparison of osmotic pressures from simulations of $\mathrm{NaCl}$ from this work (yellow) and by Luo and Roux (black, ref. 47).

Figure S3 Controls for System Size, Simulation Time, and Enantiomeric Composition. A. Scatterplot comparing osmotic coefficients obtained with different system sizes (1X or 3X volume) for Gly (circles) and Thr (triangles) in TIP3P (blue) and TIP4PEw (red) water at different concentrations. B. Osmotic coefficients obtained from 300 ns simulations of 2 M Gly in TIP3P (blue) or TIP4P-Ew (red) water averaged over three $100 \mathrm{~ns}$ segments. C. Osmotic coefficients obtained from simulations of $2 \mathrm{M}$ Ala using different molar ratios of D- and L-stereoisomers ranging from all $\mathrm{D}(0.00)$ to all L (1.00).

Figure S4 Control for Dependence on External Reference Pressure. Force output (A), solute concentration (B), and osmotic coefficient $(\mathbf{C})$ as a function of applied external reference pressure for simulations of $2 \mathrm{M}$ Thr in TIP3P water.

Figure S5 Upper Limit for Solute Concentrations from Experimental Osmotic Coefficient Determinations. Maximum molal concentrations reported for experimentally derived osmotic coefficients for the amino acids.

Figure S6 Average Errors in Simulated Osmotic Coefficients. Average values of the standard deviations of osmotic coefficients of amino acids measured at different solute concentrations in either TIP3P (blue) or TIP4P-Ew (red) water. Error bars 
indicate the standard deviations of the 16 standard deviations. The standard deviations were consistently lower at higher solute concentrations, and consistently lower when using TIP3P water.

Figure S7 Osmotic Coefficients Using TIP3P Water. Plot of osmotic coefficients for 16 amino acids in TIP3P water with respect to solute concentration. Small aliphatic residues are shown in blue, large aliphatic residues and aromatics in green, and polar residues in red. Error bars are standard deviations obtained from dividing data into 5 contiguous blocks of 20 ns.

Figure S8 Osmotic Coefficients from Simulation Compared to Experimental Results. A. Comparison of osmotic coefficients from simulation (y-axis) with experiment $(\mathrm{x}-$ axis) at $0.5 \mathrm{M}$ in TIP3P water. The black line represents a 1:1 correspondence. The colored line is the correlation for our data compared to experiment with the corresponding $\mathrm{R}^{2}$ labeled. Error bars are shown for simulation data only. B. Same as A but showing results for $1 \mathrm{M}$. C. Same as A but showing results for TIP4P-Ew water. D. Same as $\mathrm{C}$ but showing results for $1 \mathrm{M}$.

Figure S9 Osmotic Coefficients for Mixed Solutions of Glutamate + Lysine. A. Osmotic coefficients for simulations of Glu-Lys mixtures (light squares) in TIP3P water plotted versus solute concentration. Osmotic coefficients for simulations of individual Glu (circles) or Lys (triangles) that were in the best agreement with experiment are shown for reference. B. Same as A but showing results for TIP4PEw water.

Figure S10 Osmotic Coefficients Using Different Force Fields at Different Solute Concentrations. A. Comparisons of osmotic coefficients from simulations of five amino acids (Pro, Gly, Thr, Gln, and Phe) at solute concentrations of $0.5 \mathrm{M}$ using a variety of force field combinations. B. Same as A but showing results for solute concentrations of $1 \mathrm{M}$. 


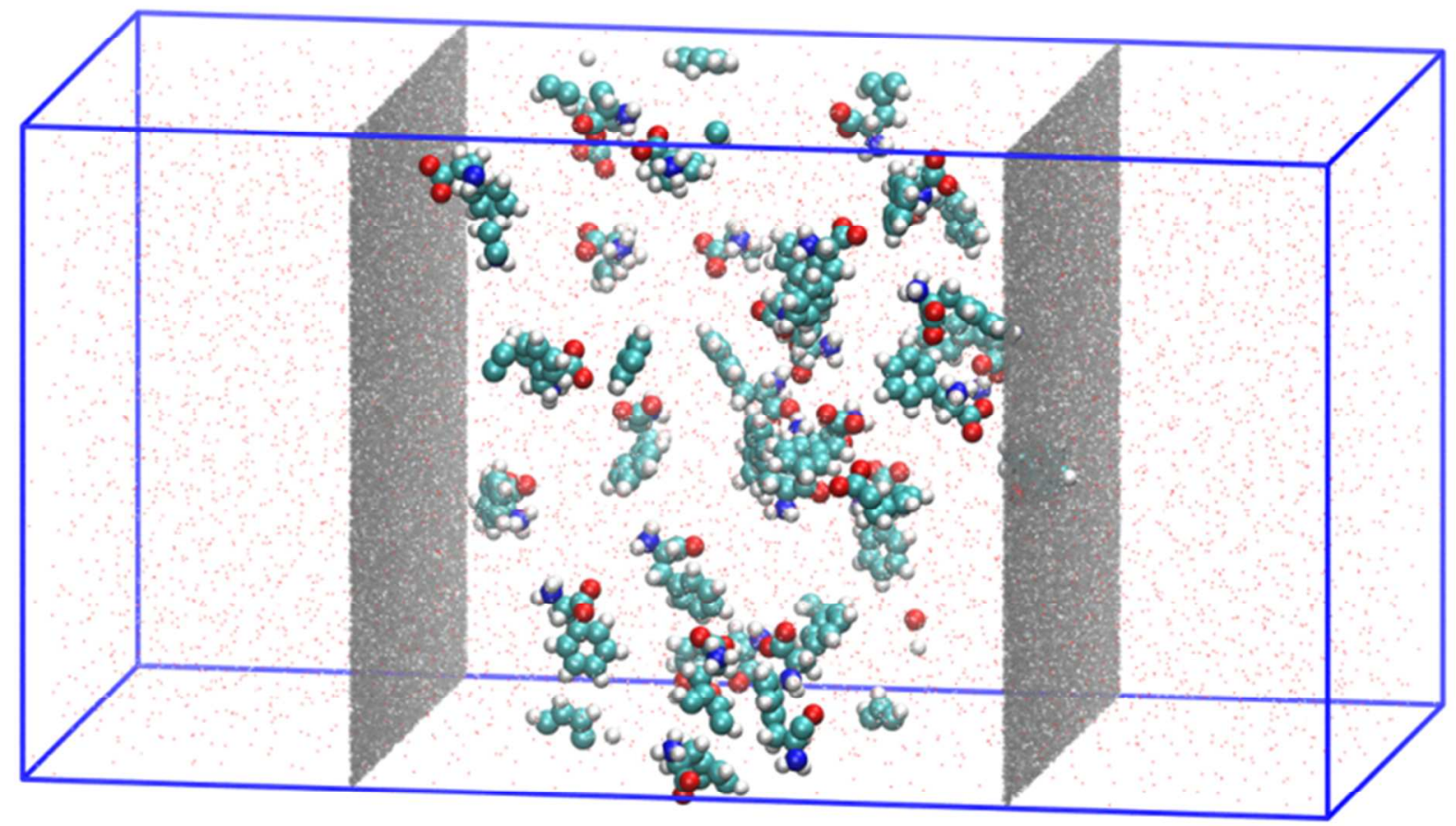

Figure S1 

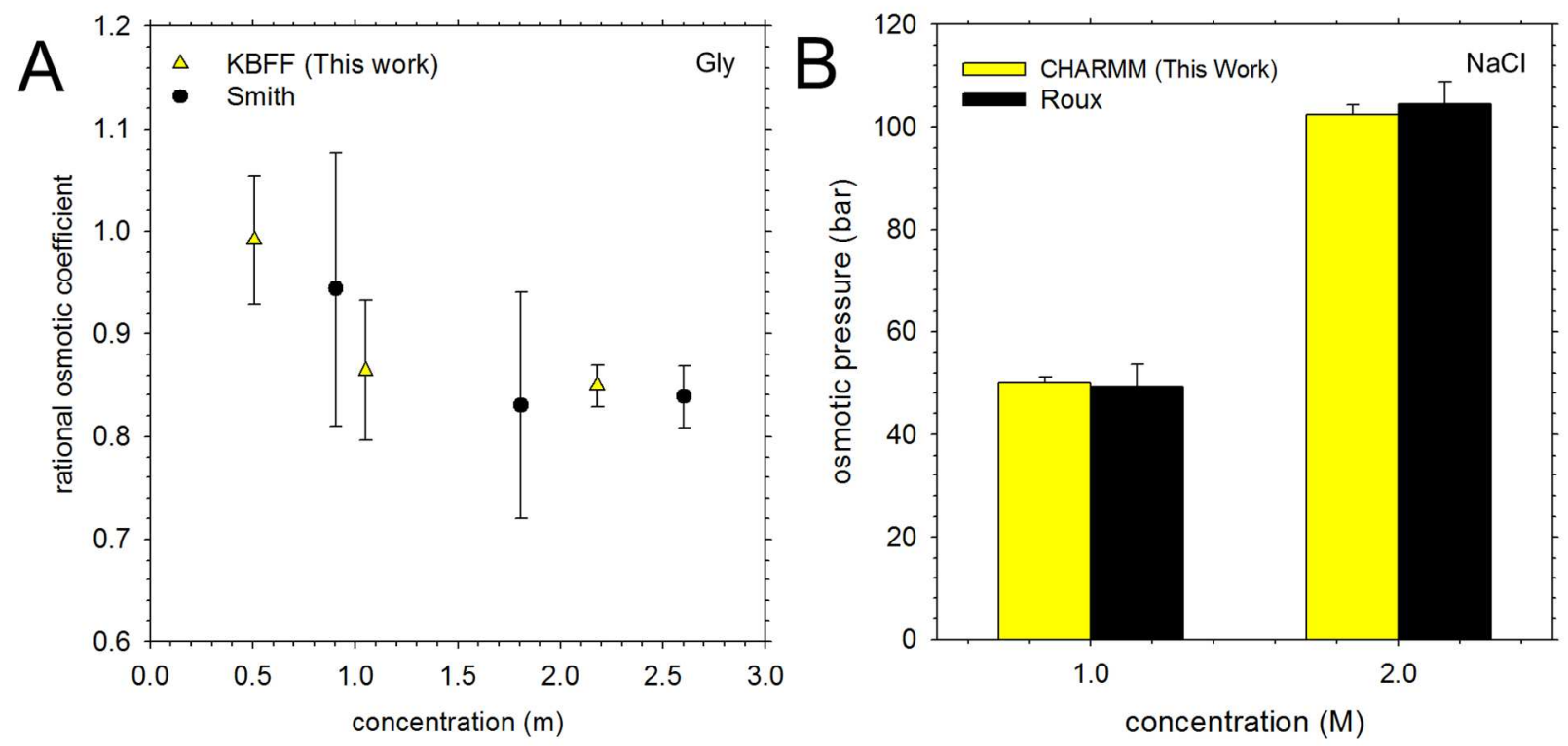

Figure S2 

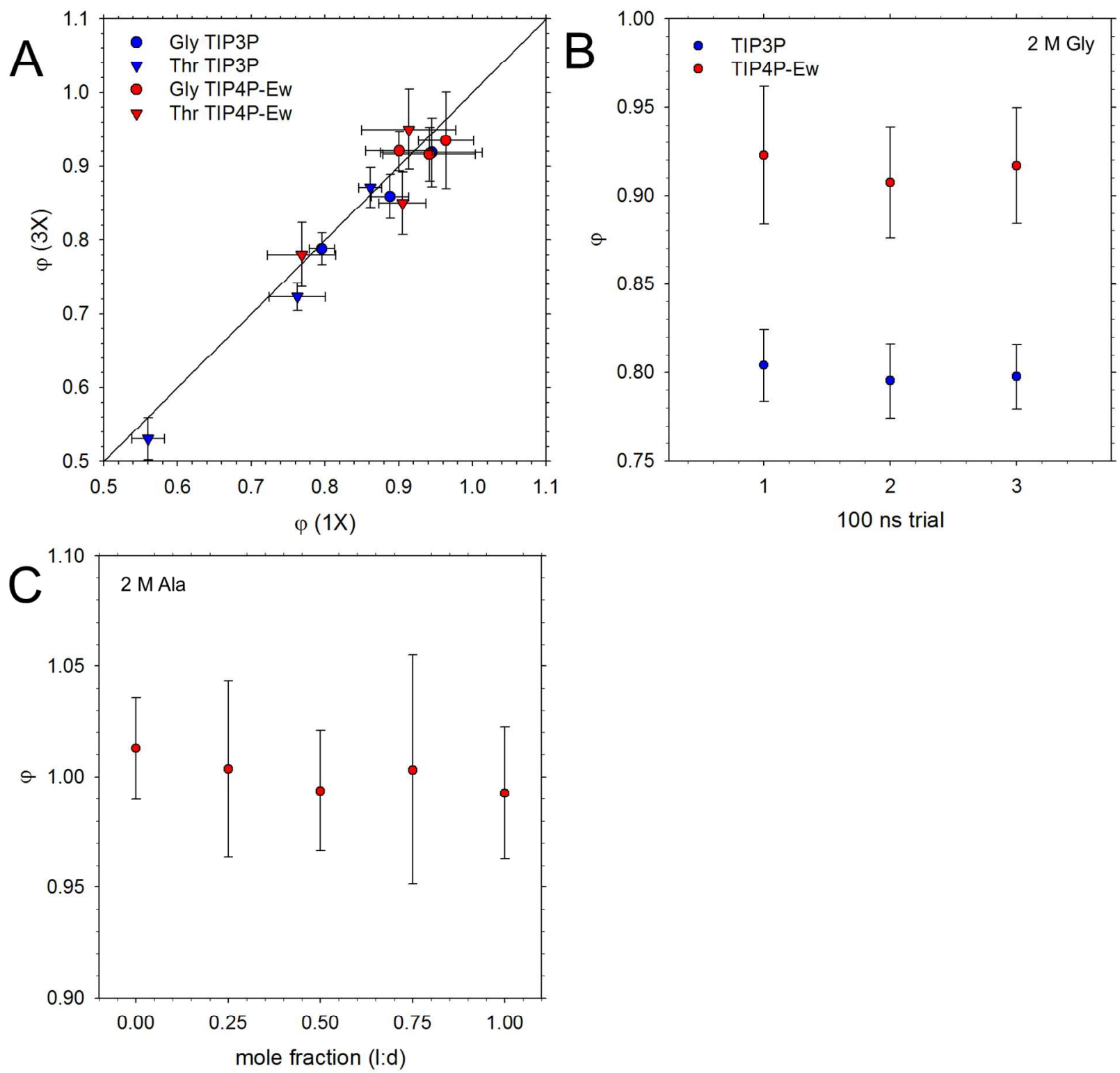

Figure S3 

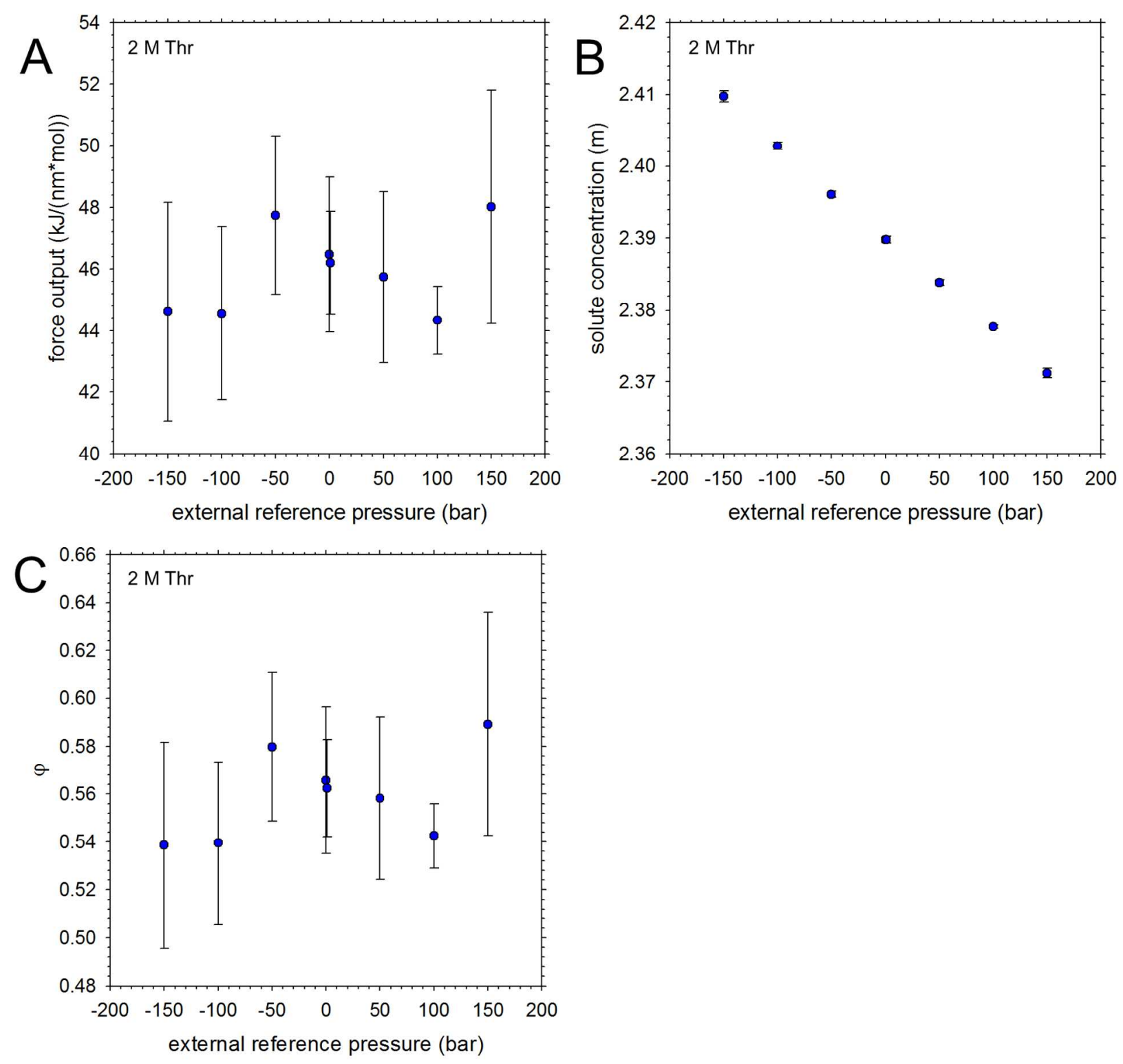

Figure S4 


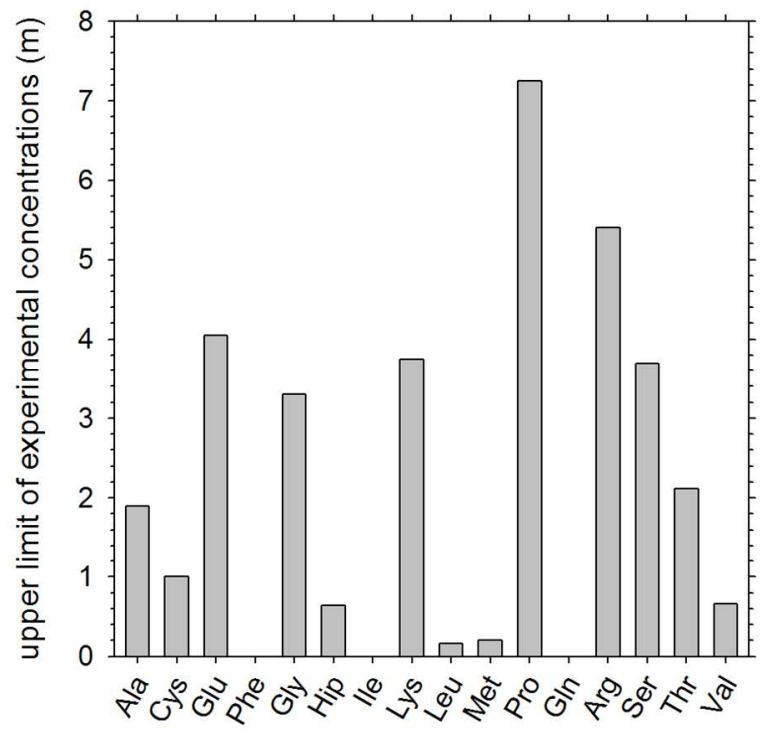

Figure S5 


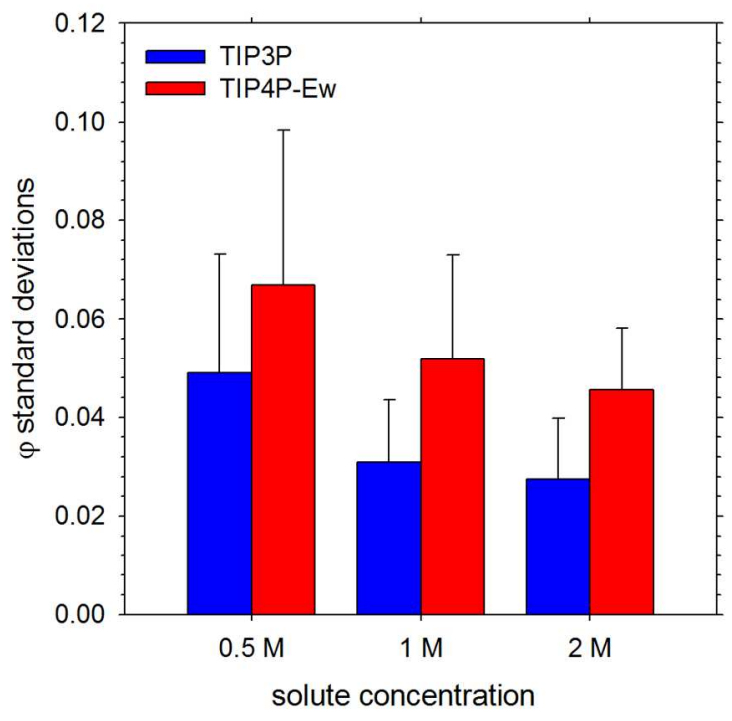

Figure S6 


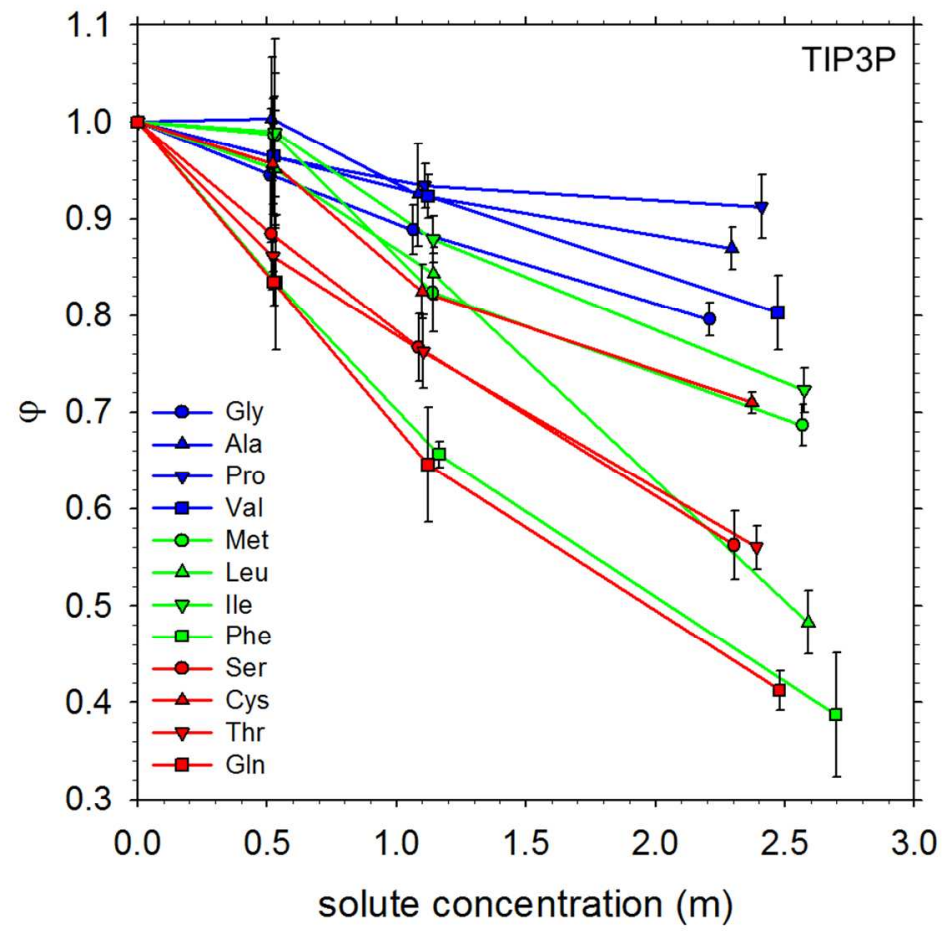

Figure S7 

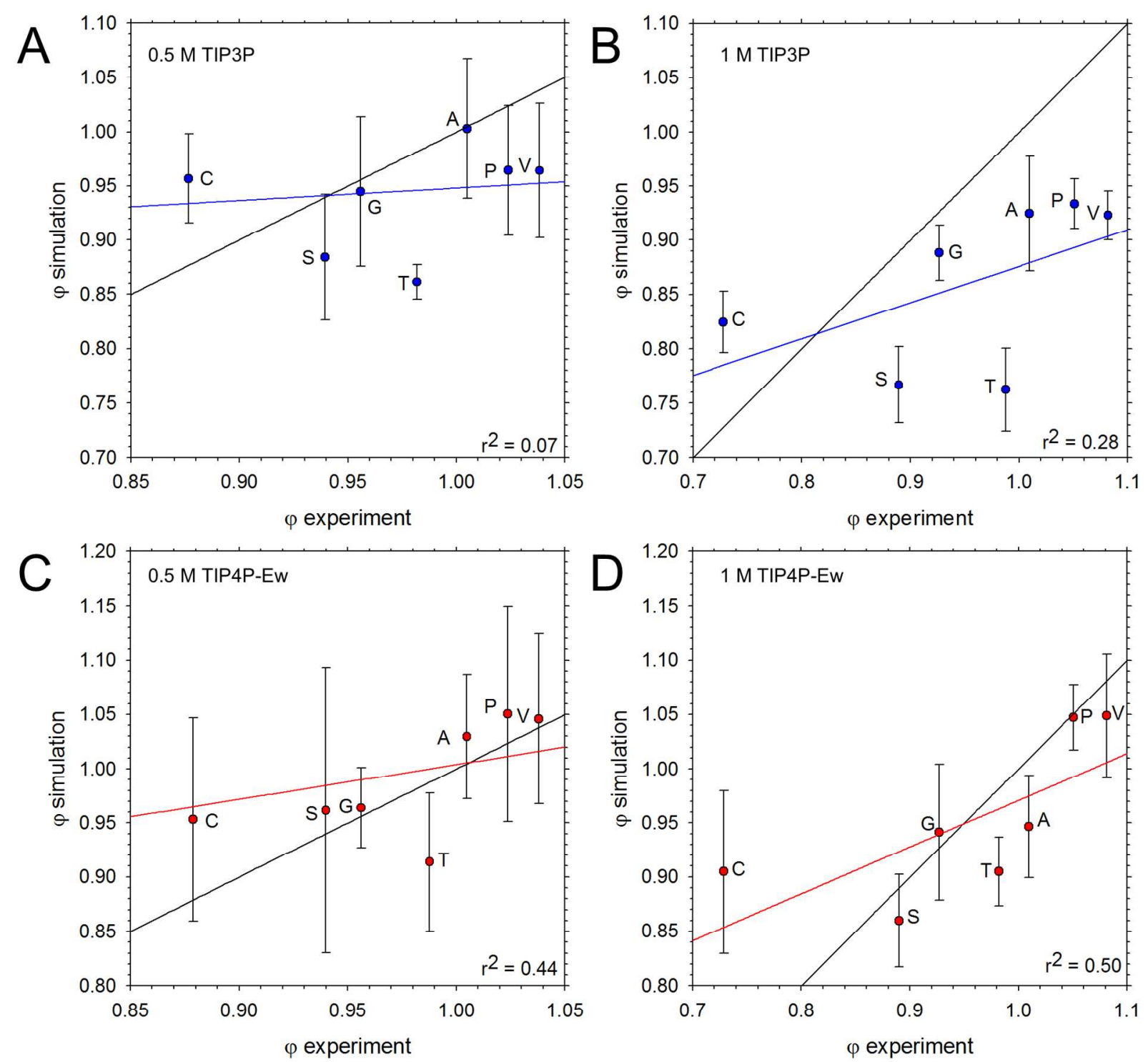

Figure S8 

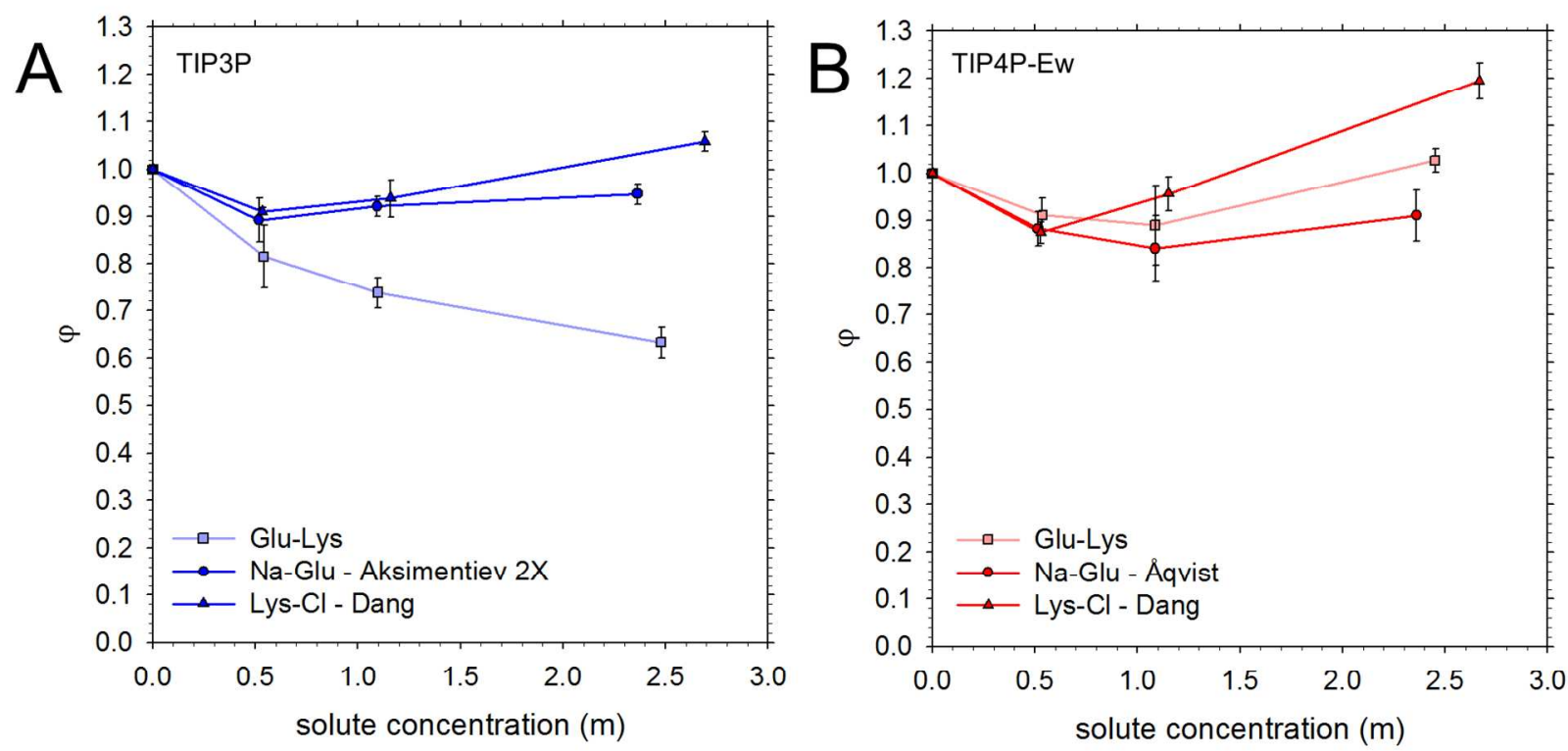

Figure S9 

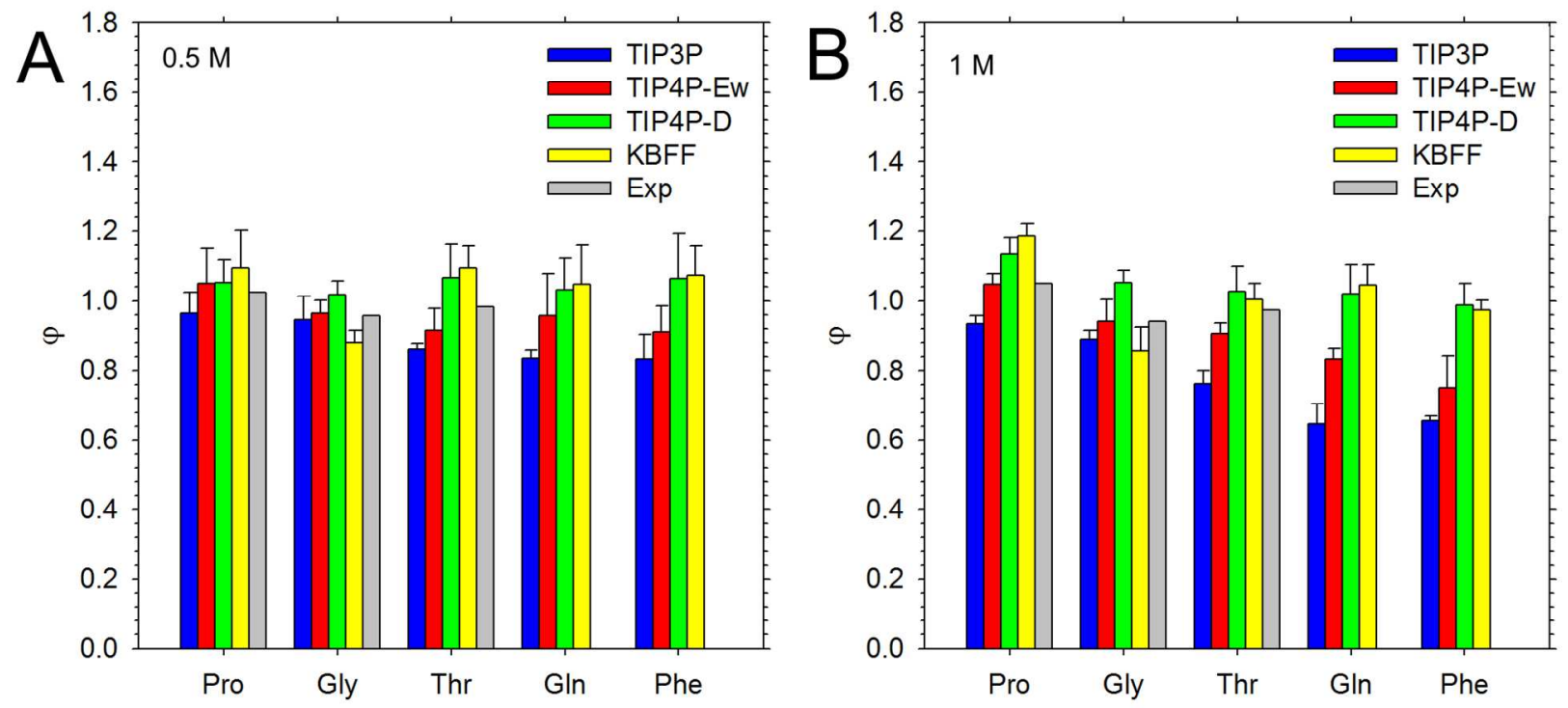

Figure S10 


\section{Files used for the re-derivation of partial charges for two-residue peptides:}

The following files for Ala-Ala (AA), Ala-Gly (AG), Gly-Ala (GA), and Gly-Gly (GG) were used in the derivation of partial charges: GAMESS input files for geometry optimization , RESP input files (two each, for the two stage fitting), and the final residue topology with charges, as implemented in the aminoacids.rtp file in GROMACS. For this last file, molecule types are designated by whether they are the $\mathrm{N}$ - or $\mathrm{C}$ terminus, followed by a two-letter designation for the two-residue peptide they represent. Thus, the C-terminal half of the peptide Ala-Gly would be called CAG. Atom types, bonds, and dihedrals are identical to those from the AMBER force field.

Supplemental files are:

AA_GEOM_OPT (GAMESS input file)

AA_RESP_INP_1 (round one RESP input)

AA_RESP_INP_2 (round two RESP input)

AA_RESIDUE_TYPE (GROMACS topology file)

AG_GEOM_OPT

AG_RESP_INP_1

AG_RESP_INP_2

AG_RESIDUE_TYPE

GA_GEOM_OPT

GA_RESP_INP_1

GA_RESP_INP_2

GA_RESIDUE_TYPE

GG_GEOM_OPT

GG_RESP_INP_1

GG_RESP_INP_2

GG_RESIDUE_TYPE 